\title{
RECONSIDERING TRIGONOMETRIC INTEGRATORS
}

\author{
DION R. J. O’NEALE ${ }^{\otimes 1}$ and ROBERT I. MCLACHLAN ${ }^{1}$
}

(Received 16 November, 2007; revised 3 December, 2008)

\begin{abstract}
In this paper we look at the performance of trigonometric integrators applied to highly oscillatory differential equations. It is widely known that some of the trigonometric integrators suffer from low-order resonances for particular step sizes. We show here that, in general, trigonometric integrators also suffer from higher-order resonances which can lead to loss of nonlinear stability. We illustrate this with the Fermi-Pasta-Ulam problem, a highly oscillatory Hamiltonian system. We also show that in some cases trigonometric integrators preserve invariant or adiabatic quantities but at the wrong values. We use statistical properties such as time averages to further evaluate the performance of the trigonometric methods and compare the performance with that of the mid-point rule.
\end{abstract}

2000 Mathematics subject classification: primary 65L05; secondary 65P10, 65P40. Keywords and phrases: numerical integration, initial value problems, highly oscillatory Hamiltonian systems, trigonometric integrators, symplectic algorithms.

\section{Introduction}

Trigonometric integrators, or exponential integrators, have enjoyed recent popularity due to their efficiency at solving highly oscillatory differential equations [4, 6, 8-10]. For a particular class of second-order differential equations with (highly) oscillatory solutions these methods require a new complete function evaluation only after a time step of one, or even many, periods of the fastest oscillations of the system.

It is already known that such methods can suffer from low-order resonances for particular step sizes but, to our knowledge, there has been little analysis of the effect of higher-order resonances for such methods. These resonances can lead to growth of errors in conserved quantities such as the total energy of Hamiltonian systems and to loss of nonlinear stability.

\footnotetext{
${ }^{1}$ Institute of Fundamental Sciences, Massey University, Private Bag 11-222, Palmerston North, New Zealand; e-mail: d.r.oneale@massey.ac.nz,r.mclachlan@massey.ac.nz.

(C) Australian Mathematical Society 2009, Serial-fee code 1446-1811/2009\$16.00
} 
The methods arise from the discretization of the differential equation

$$
\ddot{x}+\Omega^{2} x=g(x) \text { where } \Omega=\left(\begin{array}{cc}
0 & 0 \\
0 & \omega I
\end{array}\right), \quad \omega \gg 1,
$$

with blocks of arbitrary dimension and where $g(x)=\nabla U(x)$.

Partitioning $x$ as $\left(x_{1}, x_{2}\right)$ according to the blocks of $\Omega$ we see that System (1.1) is Hamiltonian; total energy is given by

$$
H(x, \dot{x})=\frac{1}{2}\left(\left|\dot{x}_{1}\right|^{2}+\left|\dot{x}_{2}\right|^{2}\right)+\frac{1}{2} \omega^{2}\left|x_{2}\right|^{2}+U(x) .
$$

The system also has an adiabatic invariant, or almost conserved quantity, of the oscillatory energy:

$$
I(x, \dot{x})=\frac{1}{2}\left|\dot{x}_{2}\right|^{2}+\frac{1}{2} \omega^{2}\left|x_{2}\right|^{2} .
$$

Preservation of these two quantities is one of the many possible criteria which one could use for judging the performance of a numerical integrator.

In Section 2 we describe one class of trigonometric integrator along with the celebrated Fermi-Pasta-Ulam (FPU) problem-a standard test problem of integrators for highly oscillatory problems. In Section 3 we illustrate the problem of higher-order resonance for trigonometric integrators using conservation of an invariant quantity (the total energy) and an adiabatic invariant (oscillatory energy) as measures of performance. In Section 4 we extend our comparison to other measures of performance and look at how the integrators treat some of the statistical properties of the FPU system. We summarize our findings in Section 5.

\section{Numerical integrators and a highly oscillatory test problem}

In this section we describe the trigonometric integrators along with the FPU problem which we will use to illustrate our results.

Applying the variation-of-constants formula to (1.1), one can verify that

$$
\begin{aligned}
\left(\begin{array}{c}
x(t) \\
\dot{x}(t)
\end{array}\right)= & \left(\begin{array}{cc}
\cos (t \Omega) & \Omega^{-1} \sin (t \Omega) \\
-\Omega \sin (t \Omega) & \cos (t \Omega)
\end{array}\right)\left(\begin{array}{l}
x_{0} \\
\dot{x}_{0}
\end{array}\right) \\
& +\int_{0}^{t}\left(\begin{array}{c}
\Omega^{-1} \sin ((t-s) \Omega) \\
\cos ((t-s) \Omega)
\end{array}\right) g(x(s)) d s
\end{aligned}
$$

is a solution, where $x_{0}$ and $\dot{x}_{0}$ are given at $t=t_{0}=0$.

An explicit, one-step, discretization is given by

$$
\begin{aligned}
& x_{n+1}=\cos (h \Omega) x_{n}+\Omega^{-1} \sin (h \Omega) \dot{x}_{n}+\frac{1}{2} h^{2} \Psi g_{n}, \\
& \dot{x}_{n+1}=-\Omega \sin (h \Omega) x_{n}+\cos (h \Omega) \dot{x}_{n}+\frac{1}{2} h\left(\Psi_{0} g_{n}+\Psi_{1} g_{n+1}\right),
\end{aligned}
$$

with constant step size $h$ and where $g_{n}=g\left(\Phi x_{n}\right), \Phi=\phi(h \Omega), \Psi=\psi(h \Omega), \Psi_{0}=$ $\psi_{0}(h \Omega)$, and $\Psi_{1}=\psi_{1}(h \Omega)$. Here $\phi(\xi), \psi(\xi), \psi_{0}(\xi)$ and $\psi_{1}(\xi)$ are real, even functions and $\psi(0)=\phi(0)=1$. This discretization is exact when $g(x)$, the nonlinear part of the differential equation, vanishes.

We omit, for brevity, further details on the design and analysis of trigonometric integrators and refer the reader to $[4,6,8,9]$. We mention, however, that the method 
TABLE 1. Filter functions for the various trigonometric integrators.

\begin{tabular}{llll}
\hline & $\psi(\xi)$ & $\phi(\xi)$ & Reference \\
\hline (A) & $\operatorname{sinc}^{2}(\xi / 2)$ & 1 & {$[5]$} \\
(B) & $\operatorname{sinc}(\xi)$ & 1 & {$[3]$} \\
(C) & $\operatorname{sinc}^{2}(\xi)$ & $\operatorname{sinc}(\xi)$ & {$[4]$} \\
(D) & $\operatorname{sinc}^{2}(\xi / 2)$ & $\operatorname{sinc}(\xi)\left(1+\frac{1}{3} \sin ^{2}(\xi / 2)\right)$ & {$[10]$} \\
(E) & $\operatorname{sinc}^{2}(\xi)$ & 1 & {$[8]$} \\
(G) & $\operatorname{sinc}^{3}(\xi)$ & $\operatorname{sinc}(\xi)$ & {$[6]$} \\
\hline
\end{tabular}

given by (2.1) and (2.2) is symmetric (that is, exchanging $h \leftrightarrow-h$ and $n \leftrightarrow n+1$ leaves the methods unchanged), for all $g(x)$ if

$$
\psi(\xi)=\operatorname{sinc}(\xi) \psi_{1}(\xi), \quad \psi_{0}(\xi)=\cos (\xi) \psi(\xi),
$$

where $\operatorname{sinc}(\xi)=\sin (\xi) / \xi$. The methods are symplectic if and only if, in addition to $(2.3), \phi(\xi)=\psi_{1}(\xi)$ holds. In this paper we consider $\operatorname{six}^{1}$ different trigonometric methods of the form (2.1), (2.2). The filter functions which characterize the methods are given in Table 1.

One can see that of the methods in Table 1 only (B) and (C) are symplectic. For comparison we also consider the mid-point rule (MPT) and the leap-frog/Verlet method, both of which are symmetric and symplectic. Although the trigonometric methods are not symplectic in general, one can show that they preserve a modified symplectic form [9, Section XIII.11, Problem (3)]. For short time periods (that is, $T=1$ ) and small (that is, $h \rightarrow 0$ ) step size all the methods are second-order accurate in position; see [9, Section XIII.2.3]. The mid-point rule and the leap-frog method which we use for comparison to the trigonometric integrators are both symmetric, symplectic, and second-order accurate.

The main test problem we use in this paper is the FPU problem with three stiff (fast/harmonic) and four soft (nonlinear) springs as it is given in [9, Section I.5.1]. The Hamiltonian for the FPU system can be written as

$$
\begin{aligned}
H= & \frac{1}{2} \sum_{i=1}^{3}\left(y_{0, i}^{2}+y_{1, i}^{2}\right)+\frac{1}{2} \omega^{2} \sum_{i=1}^{3} x_{1, i}^{2} \\
& +\frac{1}{4}\left(\left(x_{0,1}-x_{1,1}\right)^{4}+\sum_{i=1}^{2}\left(x_{0, i+1}-x_{1, i+1}-x_{0,1}-x_{1, i}\right)^{4}+\left(x_{0,3}+x_{1,3}\right)^{4}\right),
\end{aligned}
$$

$\overline{1}$ The methods are denoted (A)-(E) and (G), with (F) omitted to avoid confusion with the method (F) of [9, Section XIII.2.2]. 
where:

- $x_{0, i}$ is the scaled displacement of $i$ th stiff spring;

- $x_{1, i}$ is the scaled expansion (or compression) of the $i$ th stiff spring;

- $y_{0, i}$ and $y_{1, i}$ are the conjugate momenta of the above.

As in [9], we use initial conditions $x_{0,1}(0)=1, \quad x_{1,1}(0)=\omega^{-1}, \quad y_{0,1}(0)=1$, $y_{1,1}(0)=1$, and all other entries are zero. That is,

$$
z(0)=\left[1,0,0, \omega^{-1}, 0,0,1,0,0,1,0,0\right]^{T}
$$

where $z=\left[x_{0, i}^{T}, x_{1, i}^{T}, y_{0, i}^{T}, y_{1, i}^{T}\right], i=1,2,3$. The Hamiltonian of the system is clearly a conserved quantity. The oscillatory energy of the $i$ th stiff spring is given by

$$
I_{j}=\frac{1}{2}\left(y_{1, j}^{2}+\omega^{2} x_{1, j}^{2}\right)
$$

the total oscillatory energy $I=\sum_{j} I_{j}$ being an adiabatic invariant.

The FPU system is chaotic, and therefore, global errors are not sensible quantities to study as indicators of the performance of the numerical methods since arbitrarily close trajectories can diverge exponentially. Instead, we use measures such as the change in the Hamiltonian and preservation of other physical properties to quantify the performance of the methods. In the following section we use changes in the total and the oscillatory energy to show the effect of resonances on the numerical integrators.

\section{Resonances, energy conservation and oscillatory energy}

The problem of resonances for numerical integrators can be most easily and dramatically illustrated for a planar Hamiltonian system.

The resonances which affect the trigonometric integrators at odd and even multiples of $\pi$ are order two and one resonances, respectively. These resonances are typically unstable (result in unbounded growth with time). The trigonometric integrators can also suffer from order three resonances; these are also typically unstable but are slower to increase than the lower-order resonances. The order three resonance occurs at $h \omega / \pi=2 / 3$ for the trigonometric integrators and at $h \omega / \pi=2 \sqrt{3} / \pi \simeq 1.1$ for the mid-point rule. Order four resonances (at $h \omega / \pi=1 / 2$ for the trigonometric methods, $h \omega / \pi=2 / \pi \simeq 0.64$ for the mid-point rule) can be either stable or unstable but typically have smaller magnitude/growth than the lower-order resonances. Higherorder resonances are generally stable [1].

The analysis in [12] shows that for a planar Hamiltonian system,

$$
H(q, p)=\frac{1}{2} p^{2}+\frac{1}{2} \omega^{2} q^{2}+\frac{1}{3} B q^{3}+\frac{1}{4} C q^{4}+\mathcal{O}\left(q^{5}\right)
$$



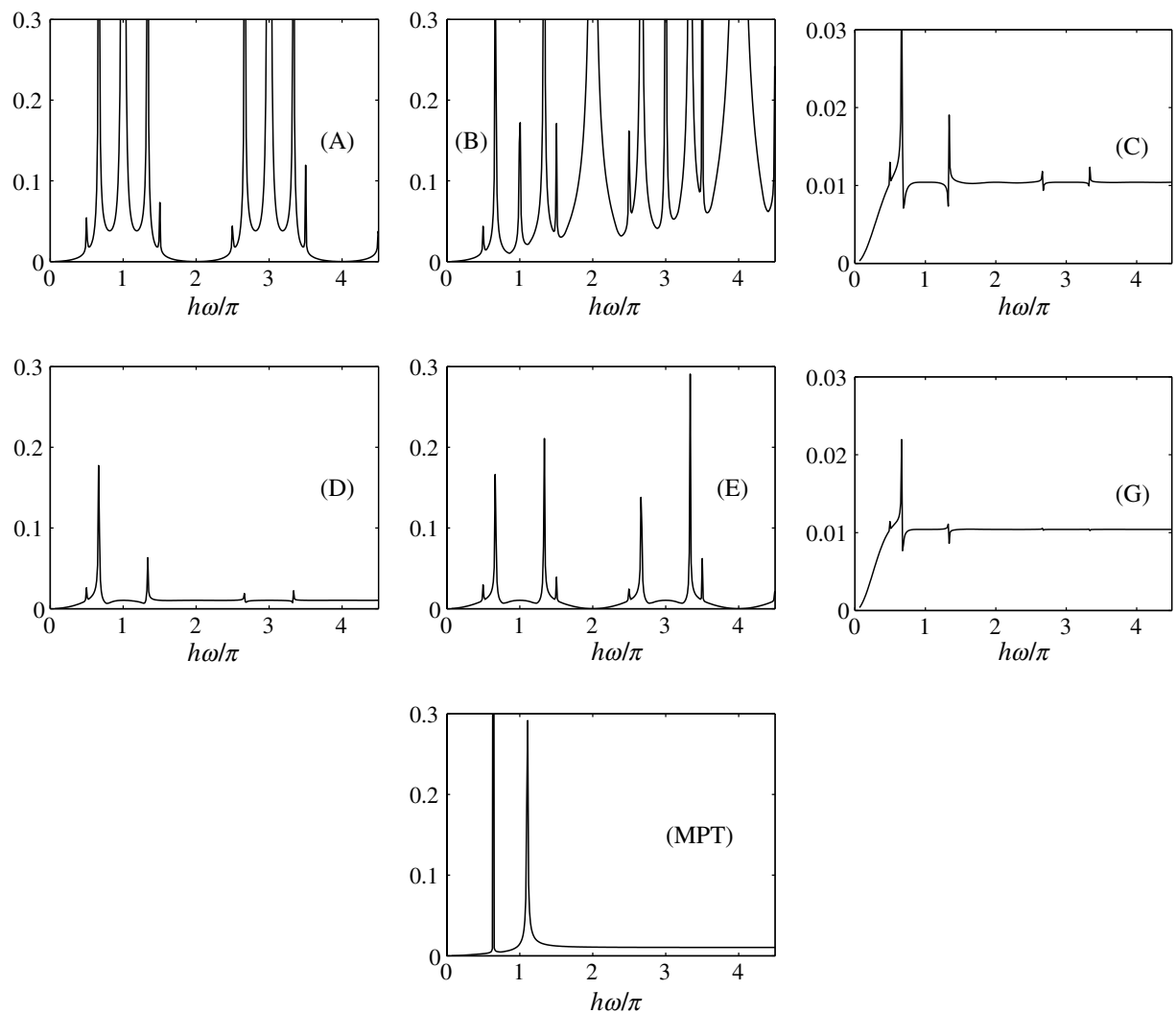

FIgURE 1. Maximum error of total energy on the interval $[0,1000]$ as a function of $h \omega / \pi$ for the Hamiltonian $H(q, p)=\frac{1}{2} p^{2}+\frac{1}{2} \omega^{2} q^{2}-\frac{1}{3} q^{3}-\frac{1}{4} q^{4}$ (step size $h=0.02$ ). Note the different vertical axis for methods $(\mathrm{C})$ and $(\mathrm{G})$.

the mid-point rule has order 3 resonances (at $h=(2 / \omega) \sqrt{3}$ ) leading to an unstable equilibrium point whenever $B \neq 0$, while the order 4 resonances $(h=2 / \omega)$ cause the equilibrium to be unstable when $\left(\omega^{2} C-B^{2}\right)\left(\omega^{2} C-2 B^{2}\right)<0$.

We take the system given by (3.1) with $B=C=-1$ and, fixing the step size at $h=0.02$, we vary $\omega$ such that $0<h \omega / \pi \leq 4.5$. Figure 1 shows the maximum deviation in the Hamiltonian after integrating the equations of motion over the interval [0, 1000].

The widths of the resonant bands for the symplectic trigonometric method (B) appear to render it unusable. Method (A) fares slightly better due to the absence of the wide resonant spikes at even multiples of $\pi$. Methods (C) and (G) seem to give excellent results, though we will see later that this is not the case in general.

The mid-point rule is not affected by resonances of order lower than three because its eigenvalues are limited to $\exp (i \theta), \theta \in(0, \pi)$. 

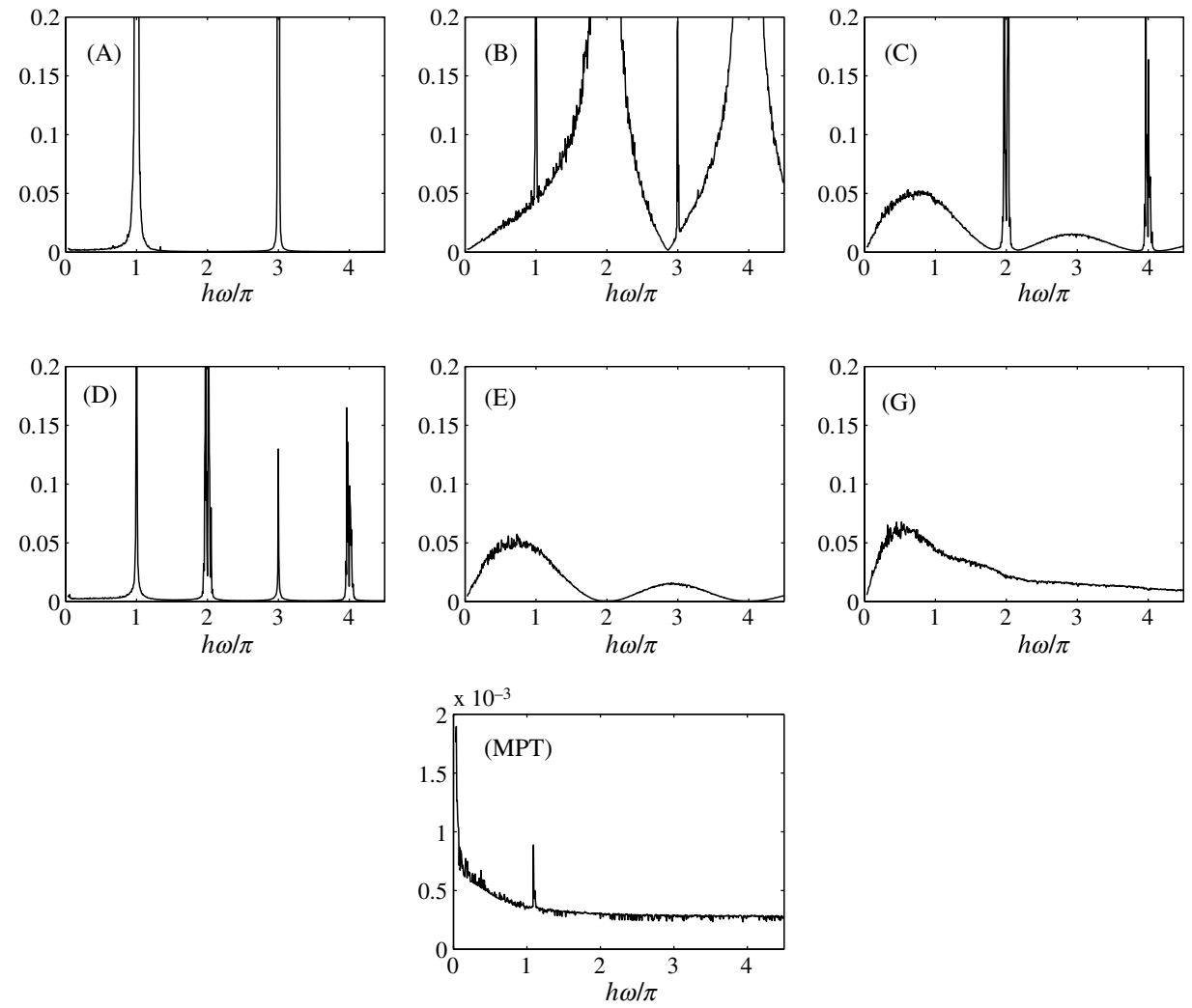

FIGURE 2. Maximum error of total energy for the FPU problem on the interval $[0,1000]$ as a function of $h \omega / \pi$ (step size $h=0.02$ ). Note the different vertical axis for (MPT).

Although the planar system considered above is sufficient to illustrate the phenomenon of resonance, it does not have the multiple time scales which make other highly oscillatory problems so challenging numerically. We turn, therefore, to the FPU problem - the standard test problem. The FPU problem is Hamiltonian - its energy is an invariant quantity. Since the system is chaotic it does not make sense to look at the global error (in position) of an individual orbit, except for very short integration lengths. Therefore, to illustrate the effect of resonances on the numerical solutions we look at the errors in the energy of numerical solutions for fixed step size and integration length. In Figure 2 we plot the maximum deviation in the energy over the interval $[0,1000]$ as a function of $h \omega / \pi$ for fixed $h=0.02$.

In Figure 2 the trigonometric methods do not appear to show any of the order three and four resonances that were so clear for the planar system. This is due to relatively slow growth of these higher-order resonances combined with the relatively short integration length. For longer integration times these resonances become apparent. We illustrate this in Figure 3 by plotting the value of the Hamiltonian for three choices 


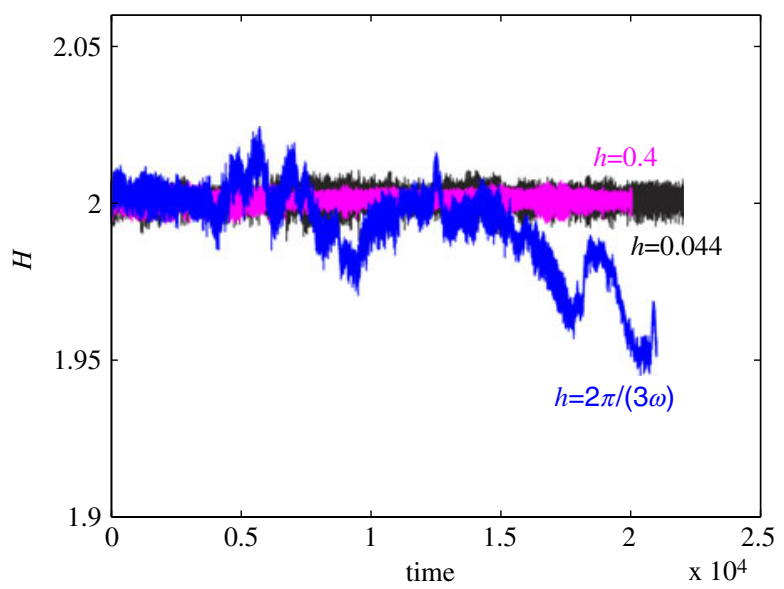

FIGURE 3. Total energy of solutions calculated with method (A) for step sizes slightly below $(h=0.04)$ and slightly above $(h=0.044)$ the resonant value of $h=2 \pi / 3 \omega \simeq 0.0419$ for $\omega=50$.

of step size using method (A) with an integration time roughly ten times longer than those used in Figure 2. We take one step size $h=2 \pi /(3 \omega) \simeq 0.0419$ on the order three resonance and two step sizes immediately either side of the resonant value, $h=0.044$ and $h=0.04$.

We observe that the maximum energy error is no longer bounded for the resonant step size.

The oscillatory energy $I=\sum_{j} I_{j}$ of the FPU system is not a conserved quantity but, rather, an adiabatic invariant: a nearly conserved quantity which oscillates about its mean value with some standard deviation. The standard deviation of $I$ is therefore possibly a better characterization of the oscillatory energy than the maximum deviation.

The exact solution of the FPU problem has $I(t)=$ Const. $+\mathcal{O}\left(\omega^{-1}\right)$. That is, the standard deviation of the total oscillatory energy as a function of $\omega$ should look like $C \omega^{-1}$. We used a very long $\left(T=10^{6}\right)$ integration period and a small $(h=$ 0.002 ) time step which resolved all the fast oscillations (for $\omega=50$ ) to calculate $\sigma_{I}$, the standard deviation of the oscillatory energy. This allowed us to determine the coefficient value $(C=0.75)$ and give a reference solution for the behaviour of $\sigma_{I}(\omega)$. Figure 4 shows $\sigma_{I} / \omega$ minus the reference solution, that is, we plot $\sigma_{I} \omega-0.75$ against $h \omega / \pi$, again for fixed step size $h=0.02$.

We can see that for the trigonometric method $(\mathrm{G})$, the cost of preventing resonances is to also prevent the correct behaviour of the oscillatory energy. The only method which manages to approximate the correct behaviour (a horizontal line at zero) is the mid-point rule. The trigonometric methods (A) and (D) show the correct behaviour away from resonant values of $\omega$. In general, for fixed $h \omega$ the value of $\sigma_{I} \omega$ does not converge to the correct value as $h \rightarrow 0$. 

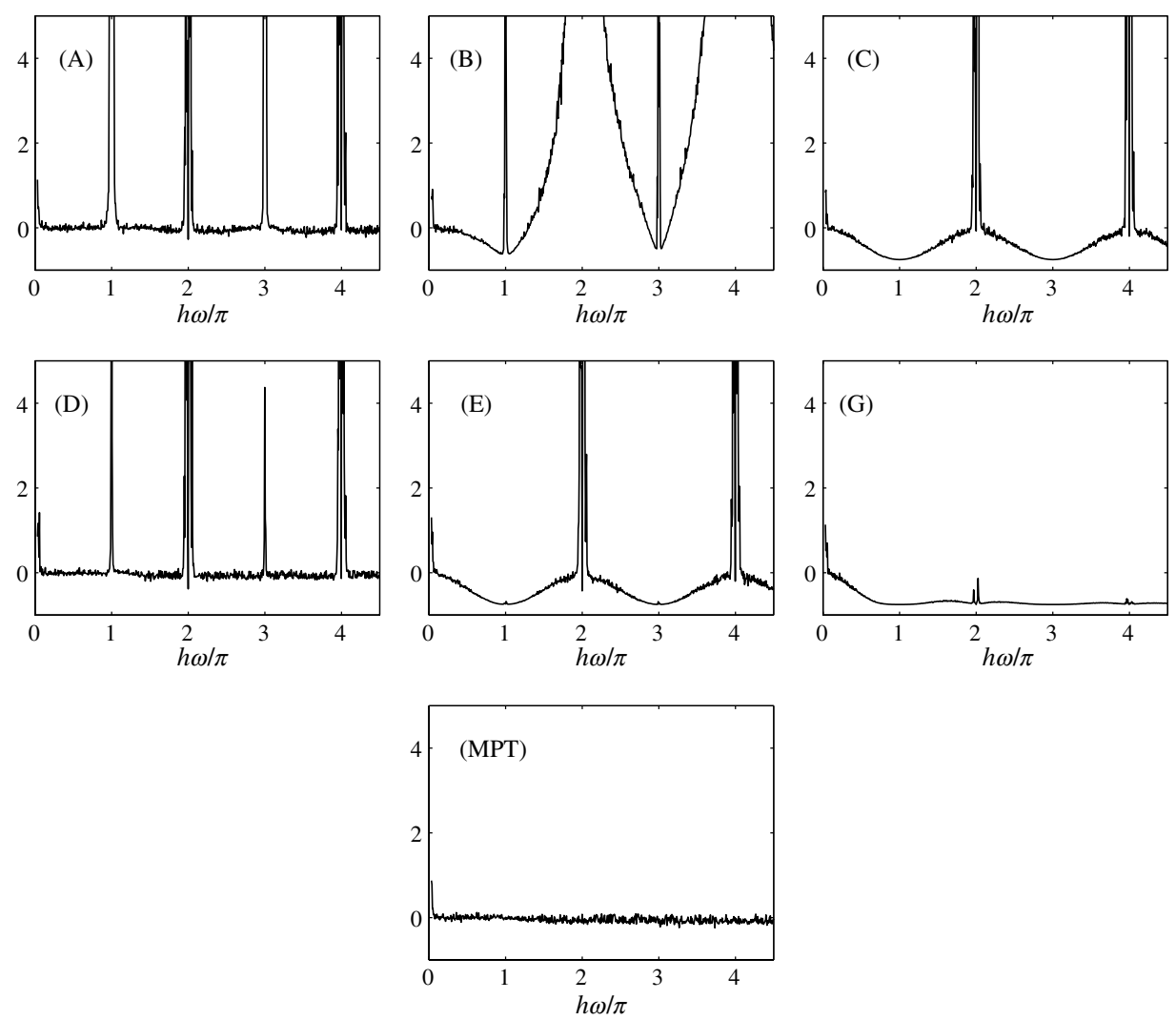

FIGURE 4. Error in the scaled standard deviation of oscillatory energy of the FPU problem, on the interval $[0,1000]$ (that is, $\left.\sigma_{I}(\omega) \omega-0.75\right)$ as a function of $h \omega / \pi$ (step size $h=0.02$ ).

Results for symplectic integrators concerning approximate energy conservation and preservation of a modified Hamiltonian hold in the limit of small step size. As the step size increases, one sees the difference between the modified Hamiltonian and the original one grow - the energy of the numerical solution oscillates with greater amplitude as step size increases and backward error analysis (the usual tool for showing near-conservation of energy) no longer holds.

It is also worth noting that although the trigonometric integrators are second-order accurate in position, they are in general only first-order accurate with respect to the total energy. That is, decreasing the step size used with the method and holding $h \omega$ fixed, one only observes a linear decrease in the error in the Hamiltonian. Exceptions are methods (A) and (D) which, along with the mid-point rule, are second-order accurate with respect to the total energy. In fact (A) and (D) satisfy a condition necessary for $\mathcal{O}\left(h^{2}\right)$ energy conservation [9, Section XIII.11, Problem (8)]. These are the same methods which showed the correct behaviour for the oscillatory energy. 


\section{Other performance measures: slow exchange and long-time statistics}

The FPU problem exhibits a slow exchange of energy between the stiff springs, that is, the distribution of $I$ between $I_{1}, I_{2}$ and $I_{3}$ changes with time. These effects take place on a time scale $t=\mathcal{O}(\omega)$. A good numerical method should capture the rate of the slow exchange and should give correct statistics for the mean distribution of energy between the stiff springs. For the trigonometric integrators to correctly approximate the slow exchange to first-order in $h$ it is necessary that their filter functions satisfy

$$
\psi(h \omega) \phi(h \omega)=\operatorname{sinc}(h \omega)
$$

(see [9, Section XIII.4.2]).

Of the trigonometric methods considered here, only (B) satisfies the slow exchange Condition (4.1), though, since the slow exchange condition is a function of $h \omega$ other methods manage to satisfy the condition for particular values of $h \omega>0$. In addition to the rate of energy exchange, a good numerical method should also ensure that the correct amount of energy is exchanged between the slow springs. This property is not guaranteed by Condition (4.1); in fact, for $h \omega \gg 0$ method (B) only transfers about $90 \%$ of the oscillatory energy between the springs, albeit on the correct time scale.

The mid-point rule, too, fails to show transfer of oscillatory energy on the correct time scale-though it fares better than the trigonometric integrators with respect to transferring all of the oscillatory energy between springs.

One is often interested in the time average of some observable rather than an individual solution. This is particularly true for chaotic systems like FPU, where accurate measurements of position are impossible due to trajectories diverging exponentially quickly, but where it may nevertheless be possible to accurately measure long-time averages. For example, symplectic integrators applied to hyperbolic Hamiltonian systems give results which converge to the correct time averages as the integration length increases independently of initial conditions (see [7, 11]).

Here we look at long-time results for the FPU problem and ask whether the numerical schemes get the correct mean values and distributions for the energy in the stiff springs. We used an integration interval of $\left[0,10^{6}\right]$ and frequency $\omega=50$ for the fast oscillations. The step size was fixed at $h=0.02$ for the trigonometric methods and the mid-point rule. As a reference solution, we used the leap-frog method with $h=0.002$, a step size small enough to resolve the fast oscillations. We saved every 100th data point for the trigonometric methods and mid-point rule and every 1000th for the leap-frog method. We present, in Table 2, the relative errors in the average values of the oscillatory energy in each of the stiff springs and the standard deviation in the total oscillatory energy using the results from the leap-frog method as a reference solution. We include also the absolute mean of the differences in $I_{j}$.

It is worth noting that for these calculations $h \omega=1$ is only moderately large; $h \omega=2.5$, for example, would give dramatically different results. 
TABLE 2. Long-time statistics for the FPU problem on the interval $\left[0,10^{6}\right]$ with $\omega=50$. Relative measurements were made with respect to the solution from the leap-frog method. The calculations with the trigonometric methods and the mid-point rule used a step size of $h=0.02$ and saved every 100 th point for the statistics. The calculation with the leap-frog method used $h=0.002$ which resolves all the fast oscillations to give a reference solution. For the leap-frog method, every 1000th point was saved.

\begin{tabular}{|c|c|c|c|c|c|}
\hline & $\frac{\bar{I}_{1}-\bar{I}_{1} \text { ref }}{\bar{I}_{2}{ }^{\text {ref }}}$ & $\frac{\bar{I}_{2}-\bar{I}_{2}{ }^{\text {ref }}}{\bar{I}_{2}{ }^{2 e f}}$ & $\frac{\bar{I}_{3}-\bar{I}_{3}{ }^{\text {ref }}}{{\overline{I_{3}}}^{\text {ref }}}$ & $\frac{\sigma_{I}-\sigma_{I}^{\text {ref }}}{\sigma_{I}^{\text {ref }}}$ & $\frac{1}{3} \sum\left|\bar{I}_{j}-\bar{I}_{j}{ }^{\mathrm{ref}}\right|$ \\
\hline (A) & $-3.11 \mathrm{e}-03$ & $1.10 \mathrm{e}-02$ & $-7.40 \mathrm{e}-03$ & $-1.46 \mathrm{e}-02$ & $2.37 \mathrm{e}-03$ \\
\hline (B) & $-3.09 \mathrm{e}-02$ & $6.86 e-02$ & $-3.51 \mathrm{e}-02$ & $-1.23 \mathrm{e}-01$ & $1.49 \mathrm{e}-02$ \\
\hline (C) & $-1.07 \mathrm{e}-02$ & $1.73 \mathrm{e}-02$ & $-6.61 \mathrm{e}-03$ & $-2.40 \mathrm{e}-01$ & $3.82 \mathrm{e}-03$ \\
\hline (D) & $1.76 \mathrm{e}-04$ & $5.88 \mathrm{e}-03$ & $-5.78 \mathrm{e}-03$ & $-1.27 \mathrm{e}-02$ & $1.31 \mathrm{e}-03$ \\
\hline (E) & $-1.72 \mathrm{e}-02$ & $4.01 \mathrm{e}-02$ & $-2.18 \mathrm{e}-02$ & $-2.50 \mathrm{e}-01$ & $8.74 \mathrm{e}-03$ \\
\hline (G) & $-5.04 \mathrm{e}-02$ & $1.09 \mathrm{e}-01$ & $-5.51 \mathrm{e}-02$ & $-3.85 \mathrm{e}-01$ & $2.37 \mathrm{e}-02$ \\
\hline (MPT) & $6.65 \mathrm{e}-03$ & $-1.41 \mathrm{e}-02$ & $6.84 \mathrm{e}-03$ & $-1.92 \mathrm{e}-02$ & $3.05 \mathrm{e}-03$ \\
\hline
\end{tabular}

The methods which give the best results for the long-time statistics for the mean difference in the oscillatory energy with respect to the reference solution are the trigonometric methods (A) and (D) and the mid-point rule - the same methods which did well at capturing the total oscillatory energy (see Section 3).

In addition to getting the average values $I_{j}$ correct, a numerical method should give the correct distribution of $I_{j}$ values, that is, it should visit the appropriate parts of the $I_{j}$ phase space for the correct amounts of time. We try to visualize this for the various methods by looking at the distribution of oscillatory energy between $I_{1}$ and $I_{2}$ (recall $\left.I_{3} \simeq 1-\left(I_{1}+I_{2}\right)\right)$, shown as a flattened three-dimensional histogram in Figure 5. We split $I_{1}$ and $I_{2}$ into 25 bins and record the amount of time that the solutions spend in each region of $I_{1}-I_{2}$ described by all possible pairs of bins. Darker regions indicate a higher frequency count for that particular bin. The reference solution shows quite a lot of structure, with certain regions of the $I_{1}-I_{2}$ plane preferred over other regions.

None of the methods considered here manage to capture all of the structure correctly, though some manage to reproduce more of the features in the plot for the reference leap-frog solution than others. For example, the reference solution spends much of its time with oscillatory energy in $I_{2}$ and $I_{3}$ but none in $I_{1}$. This feature is reproduced by the trigonometric integrator (A) but is opposite to the behaviour of the solution from $(\mathrm{G})$ and $(\mathrm{B})$. The mid-point rule manages to reproduce some but not all of the features of the reference solution.

\section{Conclusions}

We list here some of the main points from the preceding sections of this paper.

(1) Order three resonances are generally unstable and cannot be avoided; as a consequence the trigonometric integrators are unstable for $3 h \omega=2 n \pi, n \in \mathbb{Z}$. 

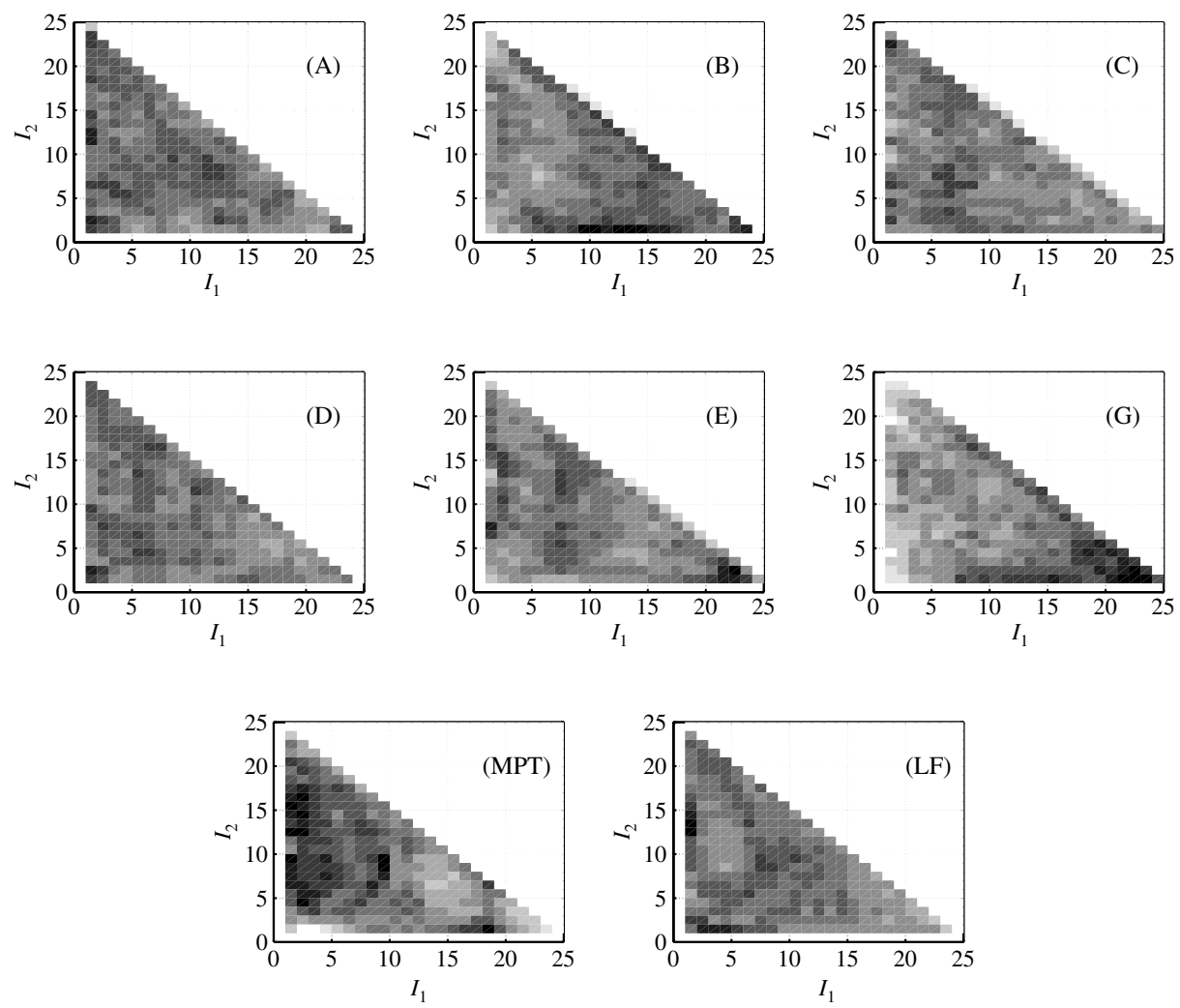

FIGURE 5. Flattened 3D histogram of amount of time spent by solutions in regions of the $I_{1}-I_{2}$ phase space. Solutions were calculated on the interval $\left[0,10^{6}\right]$ with $\omega=50$ and $h=0.02$ (every 100th point saved), except for the leap-frog (LF) solution which used $h=0.002$, to resolve all oscillations and saved every 1000 th point. Darker shading corresponds to higher frequency count.

(2) It is not enough to simply suppress resonances or to bound the variation in conserved/adiabatic quantities as this can destroy other properties of the dynamical system. Some of the trigonometric integrators show conserved quantities being preserved but at entirely the wrong value.

(3) None of the trigonometric methods manage to capture all properties and some perform worse than the mid-point rule. Although the mid-point rule is implicit while the trigonometric integrators are explicit, the cost of evaluating matrix exponentials or similar, for the trigonometric integrators, in contrast with various techniques which reduce the cost of solving the system of implicit equations, for the mid-point rule, means that the mid-point rule cannot be automatically discarded on grounds of computational cost.

Table 3 gives a comparison of the trigonometric methods and the mid-point rule for a selection of criteria. It is far from exhaustive. Other sensible measures of 
TABLE 3. A quick comparison of the performance of various methods. $H(h \omega)$ refers to the effect of resonances on the energy error (see Figures 1 and 2), while $H(h)$ refers to the energy error order behaviour of the methods for fixed $h \omega$. I refers to the preservation of the adiabatic invariant, total oscillatory energy. $d I_{j} / d t$ refers to the slow exchange of oscillatory energy between the stiff springs. A tick $(\checkmark)$ indicates that a method performs well with respect to that property; two ticks, that it performs very well; and a cross $(\times)$, that it performs poorly. The $\mathcal{O}\left(h^{n}\right)$ notation for the energy error indicates that the method has an energy error that grows like order $h^{n}$ with increasing step size.

\begin{tabular}{llllllll}
\hline & $(\mathrm{A})$ & $(\mathrm{B})$ & $(\mathrm{C})$ & $(\mathrm{D})$ & $(\mathrm{E})$ & $(\mathrm{G})$ & $(\mathrm{MPT})$ \\
\hline$H(h \omega)$ & $\checkmark$ & $\times$ & $\checkmark$ & $\checkmark$ & $\checkmark$ & $\checkmark$ & $\checkmark \checkmark$ \\
$H(h)$ & $\mathcal{O}\left(h^{2}\right)$ & $\mathcal{O}(h)$ & $\mathcal{O}(h)$ & $\mathcal{O}\left(h^{2}\right)$ & $\mathcal{O}(h)$ & $\mathcal{O}(h)$ & $\mathcal{O}\left(h^{2}\right)$ \\
$I$ & $\checkmark$ & $\times$ & $\times$ & $\checkmark$ & $\times$ & $\times$ & $\checkmark$ \\
$d I_{j} / d t$ & $\times$ & $\checkmark$ & $\times$ & $\times$ & $\times$ & $\times$ & $\times$ \\
$I_{j}$ stats & $\times$ & $\times$ & $\times$ & $\times$ & $\times$ & $\times$ & $\times$ \\
\hline
\end{tabular}

performance for highly oscillatory systems are other long-time averages, such as Lyapunov exponents — an area we will further investigate.

One possible way to achieve better results from trigonometric integrators for highly oscillatory systems is to extend the class of integrators. One possibility for this is to use more than one force evaluation per time step - for example, the method (F) of [9, Section XIII.2.2]. Another method for developing new integrators which preserve geometric properties is to use generating functions and the Hamilton-Jacobi form of the Newton equations of motion in the style of [2]. The second of these approaches seems particularly promising.

\section{References}

[1] V. I. Arnold, Mathematical methods of classical mechanics, 2nd edn, Volume 60 of Graduate Texts in Mathematics (Springer-Verlag, Berlin, 1989).

[2] C. Le Bris and F. Legoll, "Dérivation de schémas numériques symplectiques pour des systèmes hamiltoniens hautement oscillants", C. R. Acad. Sci. Paris, Ser I 344 (2007) 277-282.

[3] P. Dueflhard, "A study of extrapolation methods based on multistep schemes without parasitic solutions", Z. Angew. Math. Phys. 30 (1979) 177-189.

[4] B. García-Archilla, J. M. Sanz-Serna and R. D. Skeel, "Long-time-step methods for oscillatory differential equations", SIAM J. Sci. Comput. 20 (1998) 930-963.

[5] W. Gautschi, "Numerical integration of ordinary differential equations based on trigonometric polynomials", Numer. Math. 3 (1961) 381-397.

[6] V. Grimm and M. Hochbruck, "Error analysis of exponential integrators for oscillatory secondorder differential equations", J. Phys. A: Math. Gen. 39 (2006) 5495-5507.

[7] J. Guckenheimer and P. Holmes, Nonlinear oscillations, dynamical systems and bifurcations of vector fields (Springer-Verlag, New York, 1983).

[8] E. Hairer and Ch. Lubich, "Long-time energy conservation of numerical methods for oscillatory differential equations", SIAM J. Numer. Anal. 38 (2000) 414-441.

[9] E. Hairer, Ch. Lubich and G. Wanner, Geometric numerical integration: structure-preserving algorithms for ordinary differential equations, 2nd edn (Springer-Verlag, Berlin, 2002). 
[10] M. Hochbruck and Ch. Lubich, "A Gautschi-type method for oscillatory second-order differential equations", Numer. Math. 83 (1999) 403-426.

[11] T. Sauer and J. A. York, "Rigorous verification of trajectories for the computer simulation of dynamical systems", Nonlinearity 4 (1994) 961-979.

[12] R. D. Skeel and K. Srinivas, "Nonlinear stability analysis of area-preserving integrators", SIAM J. Numer. Anal. 38 (2000) 129-148. 